\title{
Output Characterization of Constant Bit Rate Traffic in FIFO Networks
}

\author{
Yashar Ghiassi-Farrokhfal, Student Member, IEEE, Jörg Liebeherr, Fellow, IEEE
}

\begin{abstract}
We provide an analytical proof that the departure rate of a CBR flow at an overloaded link with FIFO buffers is proportional to the flow's share of the total offered load at the link. This property of FIFO scheduling was recently validated in [1] in a series of traffic measurement experiments. An extension of the analysis to a multi-node scenario shows that the output rate of a flow in a network with many overloaded FIFO switches approaches the pessimistic values given by blind multiplexing.
\end{abstract}

\section{INTRODUCTION}

$\mathbf{T}$ HE dispersion of traffic passing through a packet switch plays a central role in traffic measurement methods that seek to infer information about the residual bandwidth on a link or a network path. As an example, when two equally sized packets are transmitted back to back to some remote destination, the dispersion of these packets, i.e., the time gap between their arrival at the destination, can be used to infer the capacity of the lowest capacity link on the traversed network path [2]. More recently, the dispersion of longer sequences of probe packets, called packet trains, has been studied to obtain estimates of the available bandwidth on a link or network path, e.g., [3]. Frequently, such estimation methods employ constant bit rate (CBR) packet trains, with fixed spacing between subsequent packets, e.g., [4].

The point of departure for this note is a recent study on available bandwidth estimation which measured the packet dispersion of a CBR packet train at a First-in-First-Out (FIFO) link with cross traffic [1]. FIFO is a work-conserving scheduling algorithm where backlogged traffic is transmitted in the order of arrival. At a FIFO link with capacity $C$ that sees arrivals from a CBR packet train with rate $r_{p, i n}$ and from CBR cross traffic with rate $r_{c}$, the output rate of the packet train, $r_{p, o u t}$, was found to approximately satisfy

$$
r_{p, \text { out }}= \begin{cases}r_{p, \text { in }} & \text { if } r_{p, \text { in }} \leq C-r_{c} \\ \frac{r_{p, \text { in }}}{r_{p, \text { in }}+r_{c}} C & \text { if } r_{p, \text { in }}>C-r_{c} .\end{cases}
$$

Thus, the output rate of probe traffic at an overloaded FIFO link is a fraction of the link capacity that is proportional to its share of the total offered load. In [1], Eq. (1) was validated using measurements of packet-level traffic in a testbed network. While this property of FIFO links may appear intuitive, a formal proof of Eq. (1) does not appear to exist in the literature.

Y. Ghiassi-Farrokhfal (yashar@comm.utoronto.ca) and J. Liebeherr (jorg@comm.utoronto.ca) are with the Dept. ECE, University of Toronto, Toronto, ON, Canada M5S 3G4. This research is supported in part by the Natural Sciences and Engineering Research Council of Canada (NSERC) and the National Science Foundation (NSF CNS-0435061).
The relevance of Eq. (1) becomes evident, when we study its implications. For example, we can use the equation to show that service curves appearing in the network calculus [5] are unable to accurately characterize FIFO scheduling. Further, when applied to a multi-node network, Eq. (1) implies that the service of a flow in a FIFO network may asymptotically degrade to that experienced by a low-priority flow.

Prior work on FIFO networks with CBR traffic is discussed in [6], [7]. For more general traffic, research on networks with FIFO scheduling has derived worst-case bounds on delays and other metrics, but has generally not considered overloaded links. For example, an analysis of FIFO scheduling for general deterministically bounded traffic is given in [5]. End-to-end delays for FIFO networks with leaky-bucket shaped traffic are derived in [8].

The rest of the paper is organized as follows. In Section II we present our system model. In Section III we formulate and prove the output characterization of CBR traffic at a single FIFO link. We extend the output characterization to multiple links in Section IV and study its asymptotic behavior. In Section V we present brief conclusions.

\section{SySTEM MODEL}

We study the performance of (through) traffic that traverses a sequence of $N$ nodes as shown in Figure 1. At each node, through traffic is statistically multiplexed with cross traffic at a buffered link with a fixed-rate capacity $C$. Links are workconserving in the sense that they transmit at rate $C$ whenever there is traffic to be transmitted. We do not consider losses due to buffer overflows, by assuming that buffers have infinite size. For the purposes of this study, we consider a fluid flow traffic model, where arrivals and departures are represented as continuous functions. Through and cross traffic may consist of a single traffic flow or an aggregate of flows.

The study applies the traffic representation of the network calculus [5], where arrivals and departures are represented by non-negative non-decreasing functions. We denote by $A_{0, n}(t)$ and $D_{0, n}(t)$ the arrivals and departures, respectively, of the through traffic at the $n$-th node in the time interval $(0, t]$ with $t \geq 0$, with $A_{0, n}(0)=D_{0, n}(0)=0$ and $A_{0, n}(t) \geq D_{0, n}(t)$ for all $t \geq 0$. Arrival and departures of cross traffic at the $n$-th node are denoted by $A_{c, n}(t)$ and $D_{c, n}(t)$. The total arrival and departure traffic at the $n$-th node are denoted by $A_{t o t, n}(t)=$ $A_{0, n}(t)+A_{c, n}(t)$ and $D_{t o t, n}(t)=D_{0, n}(t)+D_{c, n}(t)$, respectively. For the through traffic we have in addition that $D_{0, n}(t)=A_{0, n+1}(t)$ for $n<N$.

In the network calculus, the input-output relationship of traffic at a node is characterized by non-negative non-decreasing 


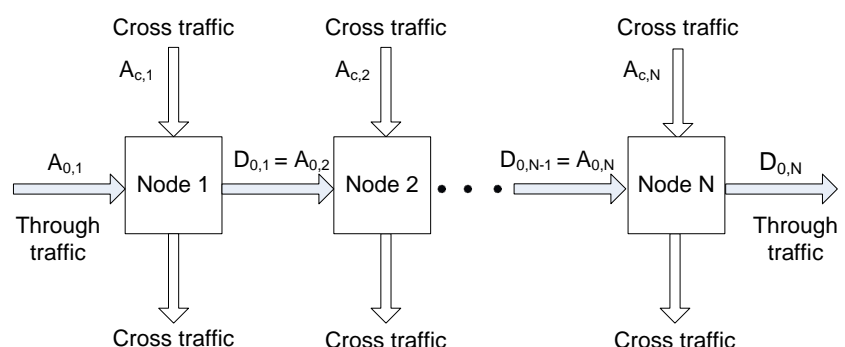

Fig. 1. Network of nodes with through traffic and cross traffic.

functions, called service curves. A function $S_{0, n}$ that satisfies

$$
D_{0, n}(t) \geq \inf _{s \geq 0}\left\{S_{0, n}(s)+A_{0, n}(t-s)\right\}, \quad \forall t \geq 0,
$$

is a (lower) service curve of the through traffic at the $n$-th node [5]. A service curve expresses service guarantees as a linear combination in a min-plus algebra, i.e., an algebra where the conventional addition is replaced by a point-wise minimization and the conventional multiplication is replaced by an addition.

\section{Single-Node OUtPut Characterization}

Theorem 1 states the correctness of the output characterization for CBR traffic at a FIFO link from Eq. (1) in terms of our system model. Since we consider only a single node in this section, we drop the subscript ' $n$ ' for arrival and departure functions.

Theorem 1: Consider two CBR flows (or flow aggregates) sharing a workconserving FIFO link with capacity $C$ and unlimited buffer size. Let $A_{0}(t)=r_{0} t$ and $A_{c}(t)=r_{c} t$ denote the arrivals of the through traffic and the cross traffic with $r_{0}, r_{c} \geq 0$. Then, the departures $D_{0}$ are characterized for all $t \geq 0$ by

$$
D_{0}(t)= \begin{cases}r_{0} t & \text { if } C \geq r_{0}+r_{c} \\ \frac{r_{0}}{r_{0}+r_{c}} C t & \text { if } C<r_{0}+r_{c} .\end{cases}
$$

Proof: Fix $t>0$ and define $t-\hat{x} \leq t$ as the beginning of the busy period containing time $t$. In other words, $t-\hat{x}$ is the last time before or at time $t$ when all arrivals have departed the link, that is,

$$
t-\hat{x}=\sup \left\{v \leq t \mid A_{t o t}(v)=D_{t o t}(v)\right\} .
$$

By the above definition, we have $D_{0}(t-\hat{x})=A_{0}(t-\hat{x})$. Since the link is work-conserving, it is transmitting in the interval $[t-\hat{x}, t]$ with rate $C$, yielding

$$
C \hat{x}=D_{t o t}(t)-D_{t o t}(t-\hat{x}) .
$$

To derive the departure characterization, we now distinguish two cases.

Case 1: $C \geq r_{0}+r_{c}$. We derive as follows:

$$
\begin{aligned}
D_{t o t}(t) & =A_{t o t}(t-\hat{x})+C \hat{x} \\
& \geq A_{t o t}(t-\hat{x})+\left(r_{0}+r_{c}\right) \hat{x} \\
& =A_{t o t}(t),
\end{aligned}
$$

where we use Eqs. (4) and (5) in the first line, the assumption $C \geq r_{0}+r_{c}$ in the second line, and the fact that $A_{t o t}(t)=$ $\left(r_{0}+r_{c}\right) t$ in the third line. It follows that $D_{0}(t)=r_{0} t$.
Since no assumption on FIFO scheduling was made, the characterization holds for any work-conserving scheduler in an underload condition.

Case 2: $C<r_{0}+r_{c}$. With $A_{t o t}(s)=\left(r_{0}+r_{c}\right) s$ and $D_{\text {tot }}(s) \leq C s$ for all $s \geq 0$, Eq. (4) implies that at $s=t-\hat{x}$ we have

$$
\left(r_{0}+r_{c}\right)(t-\hat{x}) \leq C(t-\hat{x}) .
$$

Since, at the same time, $C<r_{0}+r_{c}$, we have that $\hat{x}=t$.

Now, let us denote by $\hat{u}$ the last arrival time of through traffic that will depart the link by time $t$, that is

$$
\hat{u}=\sup \left\{v \leq t \mid A_{0}(v)=D_{0}(t)\right\} .
$$

Since we have a FIFO link and CBR fluid-flow arrivals, we also have that all cross traffic that arrives in $[0, \hat{u}]$ departs by time $t$. Thus, $D_{c}(t)=r_{c} \hat{u}$ and, therefore, $D_{\text {tot }}(t)=D_{c}(t)+$ $D_{0}(t)=\left(r_{0}+r_{c}\right) \hat{u}$. Combining this with Eq. (5) yields $\left(r_{c}+\right.$ $\left.r_{0}\right) \hat{u}=C t$. Rearranging the equation and using that $D_{0}(t)=$ $A_{0}(\hat{u})=r_{0} \hat{u}$ we obtain $D_{0}(t)=\frac{r_{0}}{r_{0}+r_{c}} C t$.

In the next section, we will compare the output characterization in a multi-node network with FIFO scheduling to the output of low-priority traffic with work-conserving (static) priority scheduling. With priority scheduling, low-priority traffic is transmitted only when there is no backlog from high-priority traffic. Since low priority traffic at a priority scheduler yields pessimistic bounds for the performance in terms of delay, backlog, or throughput for many work-conserving scheduling algorithm, this case is also called blind multiplexing [5]. We present the output characterization of blind multiplexing as a corollary to Theorem 1. (We point out that this characterization is known to hold, even for very general traffic arrival models [5])

Corollary 1: With the assumptions of Theorem 1, where we replace FIFO with priority scheduling, with cross traffic having high priority and through traffic having low priority, the departures of the through traffic are given for all $t \geq 0$ by

$$
D_{0}(t)= \begin{cases}r_{0} t & \text { if } C \geq r_{0}+r_{c} \\ {\left[C-r_{c}\right]_{+} t} & \text { if } C<r_{0}+r_{c}\end{cases}
$$

where we use the notation $[\operatorname{expr}]_{+}=\max \{\operatorname{expr}, 0\}$.

Proof: Fix $t \geq 0$. For $C \geq r_{0}+r_{c}$, we can use the corresponding proof in Theorem 1 since there is no assumption on FIFO scheduling. If $C<r_{0}+r_{c}$, it again holds that $\hat{x}=t$, i.e., the busy period starts at time 0 . Thus, we have $C t=$ $D_{\text {tot }}(t)=D_{c}(t)+D_{0}(t)$. If $C \leq r_{c}$, then there is always cross traffic in the buffer and no through flow will ever be served, i.e., $D_{0}(t)=0$. For $C>r_{c}$, any arrival from crosstraffic will be served immediately without buffering, that is, $A_{c}(s)=D_{c}(s)$ for all $s \leq t$, and the through flow departures are determined by the unused capacity, i.e., $D_{0}(s)=C s-$ $D_{c}(s)=\left(C-r_{c}\right) s$, which completes the proof.

In [9], it was suggested (without proof) that the conjecture of Eq. (1) implies a service curve $S_{0}(t)=\left[C-r_{c}\right]_{+} t$ for FIFO links with CBR traffic. The next corollary provides a proof of this and further shows that the rate of this service curve cannot be improved. This means that the service guarantees of a service curve for a FIFO link are not stronger than 
the pessimistic guarantees of blind multiplexing. This weak guarantee may appear at odds with the much stronger output guarantee given by Theorem 1. However, it merely points out a limitation in the network calculus, which is unable to accurately characterize the service at a FIFO link in terms of a min-plus linear combination as in Eq. (2). We note that generalizations of the service curve concept may be more suitable for capturing such non-linearities [10].

Corollary 2: At a FIFO link satisfying the assumptions of Theorem 1, $S_{0}(t)=\left[C-r_{c}\right]_{+} t$ is a lower service curve of the through traffic with maximal (long term) rate.

Proof: The service curve $S_{0}$ must satisfy Eq. (2) for any choice of $A_{0}(t)=r_{0} t$ and the corresponding output $D_{0}$ as given by Theorem 1 . For $C \geq r_{0}+r_{c}$, this holds since Theorem 1 gives $D_{0}(t)=r_{0} t=\inf _{s \geq 0}\left\{S_{0}(s)+r_{0}(t-s)\right\}$. For $C<r_{0}+r_{c}$, Theorem 1 gives $D_{0}(t)=\frac{r_{0} C}{r_{0}+r_{c}} t$, and $\inf _{s \geq 0}\left\{S_{0}(s)+r_{0}(t-s)\right\}=\left[C-r_{c}\right]_{+} t$; Eq. (2) then follows since $\frac{r_{0} C}{r_{0}+r_{c}} \geq\left[C-r_{c}\right]_{+}$.

Now suppose the rate is not maximal. Consider $S^{\prime}(t)=[C-$ $\left.r_{c}+\varepsilon\right]_{+}(t-\delta)$, for some $\varepsilon>0$ and some $\delta \geq 0$. $S^{\prime}$ is a lower bound for any service curve with a long term rate exceeding $\left[C-r_{c}\right]_{+}$. We can compute $\inf _{s \geq 0}\left\{S^{\prime}(s)+r_{0}(t-s)\right\}=$ $\min \left(\left[C-r_{c}\right]_{+}+\varepsilon, r_{0}\right)(t-\delta)$. When $r_{0}+r_{c}>C$, Eq. (2) thus states that

$$
\frac{r_{0} C}{r_{0}+r_{c}} t \geq \min \left\{\left[C-r_{c}\right]_{+}+\varepsilon, r_{0}\right\}(t-\delta), \quad \forall t \geq 0,
$$

Dividing by $t$ and letting $t \rightarrow \infty$, we see that any choice of $r_{0}<\left[C-r_{c}\right]_{+}+\varepsilon$ yields a contradiction.

\section{Multi-node OutPut Characterization}

We next show that, in a multi-node setting, the departure traffic passing through a sequence of FIFO queues can degrade to that observed with blind multiplexing. We assume a network as shown in Fig. 1 with $N$ nodes with fixed capacity $C$ and FIFO schedulers. Through traffic arrives to the first node as CBR fluid-flow traffic with rate $r_{0}$. At each node, we assume CBR cross-traffic with the same rate $r_{c}$. The following theorem characterizes the through traffic that departs from the last node.

Theorem 2: Under the assumptions stated above, if the number of nodes grows large $(N \rightarrow \infty)$, departures of the through traffic from the last node, denoted by $D_{0, \infty}$, are characterized for all $t \geq 0$ by

$$
D_{0, \infty}(t)= \begin{cases}r_{0} t & \text { if } C \geq r_{0}+r_{c} \\ {\left[C-r_{c}\right]_{+} t} & \text { if } C<r_{0}+r_{c} .\end{cases}
$$

Proof: If $r_{0}+r_{c} \leq C$, then by Theorem 1, the through flow arrival and departures from the first node are equal and $D_{0,1}(t)=A_{0,1}(t)=r_{0} t$. Since the arrivals to the $n$-th nodes are equal to the departures of the $(n-1)$-th node, we get $D_{0, \infty}(t)=A_{0,1}(t)=r_{0} t$.

Now we consider $r_{0}+r_{c}>C$. If $r_{c}=0$, then $D_{0, n}(t)=C t$ for all $1 \leq n \leq N$, and Eq. (7) obviously holds. For $r_{c}>0$, we will show by induction over the number of nodes $n$, that the through traffic departures from the $n$-th node $(1 \leq n \leq N)$ satisfy $D_{0, n}(t)=r_{0, n} t$, with

$$
r_{0, n}=\frac{r_{0, n-1} C}{r_{0, n-1}+r_{c}}
$$

where we set $r_{0,0}=r_{0}$ to be the arrival rate at the first node. Moreover, we will show that for all $1 \leq n \leq N$, we have that

$$
\left[C-r_{c}\right]_{+}<r_{0, n}<r_{0, n-1} .
$$

For $n=1$, the validity of Eq. (8) follows from Theorem 1 . With $r_{0}>0, r_{0}+r_{c}>C$, and $A_{0,1}(t)>D_{0,1}(t)$, we also obtain Eq. (9).

Now assume that the departure rate of the through traffic from the $k$-th node is given by $r_{0, k}=\frac{r_{0, k-1} C}{r_{0, k-1}+r_{c}}$ and that $\left[C-r_{c}\right]_{+}<r_{0, k}<r_{0, k-1}$. Recall that $D_{0, k}(t)=A_{0, k+1}(t)=$ $r_{0, k} t$. Thus, with the induction assumption $C-r_{c}<r_{0, k}$, we obtain from Theorem 1 that the departure rate is given by $r_{0, k+1}=\frac{r_{0, k} C}{r_{0, k}+r_{c}}$. Moreover, with $r_{c}>0$ we have that $r_{0, k+1}<r_{0, k}$, and since $r_{0, k}>0$ and $r_{0, k}+r_{c}>C$, by assumption, we obtain $\left[C-r_{c}\right]_{+}<r_{0, k+1}<r_{0, k}$, which completes the induction.

According to Eq. (9), the rate of through flow departures strictly decreases in $n$. Since the departure rate is also lower bounded by $\left[C-r_{c}\right]_{+}$, we obtain $r_{0, \infty}=\left[C-r_{c}\right]_{+}$.

Likewise, an iterative application of Corollary 1 yields that Theorem 2 also holds for blind multiplexing. Thus, the output rate of a CBR traffic flow or flow aggregate that traverses a long path of overloaded FIFO scheduler approaches the pessimistic rate obtained with blind multiplexing.

\section{CONCLUSION}

We analytically proved a conjecture that the departure rate of a CBR flow to a FIFO link is proportional to its share of the total offered load. Studying the implications of this property, we were able to gain new insights into FIFO scheduling. We showed that it is not feasible to give an accurate characterization of the service at a FIFO link in terms of service curves. Further, for CBR traffic arrivals we showed that the throughput under FIFO scheduling offers no improvement over blind multiplexing, when the number of nodes grows large.

\section{REFERENCES}

[1] B. Melander, M. Bjorkman, and P. Gunningberg, "First-come-firstserved packet dispersion and implications for TCP," in Proc. IEEE GLOBECOM, Nov. 2002, vol. 3, pp. 2170-2174.

[2] S. Keshav, "A control-theoretic approach to flow control," in Proc. ACM Sigcomm, 1991, pp. 3-15.

[3] C. Dovrolis, P. Ramanathan, and D. Moore, "What do packet dispersion techniques measure?," in Proc. IEEE INFOCOM, Apr. 2001, pp. 905914.

[4] B. Melander, M. Björkman, and P. Gunningberg, "A new end-to-end probing and analysis method for estimating bandwidth bottlenecks," in Proc. IEEE GLOBECOM, Nov. 2000, pp. 415-420.

[5] J. Y. Le Boudec and P. Thiran, Network Calculus, New York, NY: Springer Verlag, Lecture Notes in Computer Science, LNCS 2050, 2001.

[6] J. Sahni, P. Goyal, and H. Vin, "Scheduling CBR flows: FIFO or perflow queuing?," in Proc. 9th IEEE NOSSDAV, June 1999, pp. 13-27.

[7] J. Kaur, Scalable Network Architectures for Providing Per-flow Service Guarantees, Ph.D. thesis, Department of Computer Sciences, University of Texas at Austin, 2002.

[8] L. Lenzini, E. Mingozzi, and G. Stea, "A methodology for computing end-to-end delay bounds in FIFO-multiplexing tandems," Performance Evaluation, vol. 65, no. 11-12, pp. 922-943, 2008.

[9] J. Liebeherr, M. Fidler, and S. Valaee, "A min-plus system interpretation of bandwidth estimation," in Proc. IEEE INFOCOM, May 2007, pp. 1127-1135.

[10] R. L. Cruz and A. P. Blanc, "A service abstraction with applications to network calculus," in Proc. 41th Annual Allerton Conference on Communication, Control, and Computing, October 2003, pp. 11541163. 